\title{
Sparse detection in the chirplet transform: application to FMCW radar signals
}

\author{
Fabien Millioz and Michael Davies
}

\begin{abstract}
This paper aims to detect and characterise a signal coming from Frequency Modulation Continuous Wave radars. The radar signals are made of piecewise linear frequency modulations. The Maximum Chirplet Transform, a simplification of the chirplet transform is proposed. A detection of the relevant Maximum Chirplets is proposed based on iterative masking, an iterative detection followed by window subtraction that does not require the recomputation of the spectrum. This detection is designed to provide a sparse subset of Maximum Chirplet coefficients. The chirplets are then gathered into linear chirps whose starting time, length, and chirprate are estimated. These chirps are then gathered again back into the different Frequency Modulation Continuous Wave signals, ready to be classified. An illustration is provided on synthetic data.
\end{abstract}

Index Terms-LPI radar, FMCW radar, chirplet transform, parameter estimation

\section{INTRODUCTION}

Low Probability of Intercept (LPI) Radars are a type of radar designed to hide their emissions from hostile receivers. It aims to see without being seen, which is critical on battlefields. This goal may be achieved by several techniques, such as power management, antenna side lobe reduction or frequency agility [1].

This paper aims to detect and identify Frequency Modulation Continuous Wave (FMCW) radars. This class of LPI radar signals may be modelled by

$$
r(t)=\sum_{n=1}^{N} A_{n} \cos \left(2 \pi f_{n}(t) t+\phi_{n}\right),
$$

with $f_{n}(t)$ a piecewise linear function. In practice, some chirps may be very short with a very high or very low chirprate, and thus very difficult to handle. To extend the possibility of the model, we consider both continuous and non-continuous functions $f_{n}(t)$, to avoid the problem of very sharp chirps.

In the context of a radar interceptor, a sensor should detect a signal $s(t)$ coming from several LPI radars, embedded in a white Gaussian noise $n(t)$ of variance $\sigma^{2}$

$$
s(t)=n(t)+\sum_{i=1}^{I} r_{i}(t)
$$

where $I$ radars emit the signals $r_{i}(t)$.

Given such a model, the chirplet transform [2] seems to be a natural tool to analyse the signal and several methods already exist based on the chirplet transform. The matching-pursuit (MP) based method [3] employed by Leveau et al. searches for chirps in the signal and iteratively subtract them from the signal. This technique was applied to the detection of radar signals in [4]. The main drawback is the computations required by the matching-pursuit framework making it impractical for real time deployment. Here we are interested in a minimal computation approach. Chirplet chains [5], [6] are based on the search of a single best path in the time-frequency domain, and are applied in the low Signal-to-Noise Ratio (SNR) context of gravitational wave detection. Only one non-linear chirp is detected in the signal, consequently this approach is inappropriate for the multiple chirps of model (1). A more general chirplet chain based on a parametric model is proposed by Dugnal et al. [7], which uses local maxima to start chirplet chains, and a single criterion based on the smoothness of the frequency modulation. Other approaches to detec and estimate the parameters of chirps exist, based for example on higher order moments [8] or evolutionary algorithm [9]. However, these methods have the same computational problems than the MP methods.

For our problem, we have strong a priori information that the chirps are piece-wise linear, and in a multi-signal case, chirps may be crossing. This paper proposes a new algorithm for detecting and estimating the parameters of the chirps constituting multiple FMCW radars. This method is based on the chirplet transform, presented in the section II. An approximation of the chirplet transform is proposed, based on the difference between the signal's chirprate and the chirprate of the analysing chirplet. However, the chirplet transform has one more dimension than the usual time-frequency representation. Compared to the Short Time Fourier Transform, the additional dimension leads to more coefficients and spreads the signal's energy on more coefficients. More memory is required to deal with the chirplet transform, and more coefficients are detected as containing a part of the signal's energy. Section III proposes the Maximum Chirplet Transform, time-frequency representation based on the maximum over the chirprates of the chirplet coefficients. The spectrum of a chirp in this representation is studied, as well as the distribution of coefficients for noise only data. A detection method, based on a single chirplet transform followed by an iterative subtraction of chirp's spectrum is proposed, with the aim to detect as few relevant coefficients as possible without recomputation of the chirplet transform between the iterations.

The goal is then to amalgamate the chirplets coming from a chirplet transform into different chirps, corresponding to the instantaneous frequency lines $f_{n}(t)$ from the model (1). To do this, section IV uses the output of the detection in the Maximum Chirplet Transform. These detected coefficients are gathered into chirps, using a time-frequency-chirprate criterion. The case of chirps with a chirprate outside the range analysed by the chirplet transform is also studied.

Finally, these chirps are amalgamated back into the signals, 
using a time-frequency proximity criterion. A result of the complete algorithm is given on a synthetic signal imitating an antenna receiving four FMCW radar signals, with Signal-toNoise ratio from $-3 \mathrm{~dB}$ to $-18 \mathrm{~dB}$.

\section{Chirplet TRANSFORM}

The chirplet transform [2] $C_{x}(t, f, c)$ of a signal $x(t)$ is determined by

$$
C_{x}(t, f, c)=\int_{\tau=-\infty}^{+\infty} x(t+\tau) \phi_{T}(\tau) e^{-j 2 \pi \frac{c}{2} \tau^{2}} e^{-j 2 \pi f \tau} d \tau
$$

with $t, f$ are the time and frequency indices respectively, $\phi_{T}(\tau)$ a smoothing window of length $T$ centred on time $\tau=0$ of maximum value 1 .

The chirplet transform may be interpreted twofold. By considering a chirped window $\phi_{T, c}(\tau)=\phi_{T}(\tau) e^{j 2 \pi \frac{c}{2} \tau^{2}}$, the chirplet transform has the same definition as the usual Short Time Fourier Transform. A fast computation using a Fast Fourier Transform algorithm is thus possible. On the other hand, it may be interpreted as the projection of the signal $x(t)$ onto a set of atoms $e^{-j 2 \pi f \tau+\frac{c}{2} \tau^{2}}$, corresponding to chirplets of different chirprates $c$, centred on time $t$ and frequency $f$.

There is also a direct link to the Fractional Fourier Transform $X_{\alpha}(u)$, defined as

$$
\begin{aligned}
& X_{\alpha}(u)=\sqrt{1-j \cot \varphi} \int x(\tau) e^{j \pi\left(\tau^{2} \cot \varphi-2 u \tau \csc \varphi+u^{2} \cot \varphi\right)} d \tau \\
& =\sqrt{1-j \cot \varphi} e^{j \pi u^{2} \cot \varphi} \int x(\tau) e^{j 2 \pi \frac{\cot \varphi}{2} \tau^{2}} e^{-j 2 \pi u \tau \csc \varphi} d \tau
\end{aligned}
$$

with $\varphi=\frac{\pi \alpha}{2}$. The chirplet transform is equal to a short time fractional Fourier transform, with a chirp parameter $c=-\frac{\cot \varphi}{2}$ and the frequency $f=u \csc \varphi$, up to a factor of proportionality $\sqrt{1-j \cot \varphi} e^{j \pi u^{2} \cot \varphi}$, depending on the fractional Fourier transform parameter. Consequently, the methods proposed in this paper could also be applied to fractional Fourier Transform as well as to the chirplet transform.

\section{A. Stationary phase approximation}

Consider a single linear chirp $x(t)$

$$
x(t)=A e^{j 2 \pi\left(f_{0} t+\frac{c_{0}}{2} t^{2}\right)} .
$$

Its chirplet transform is

$$
\begin{aligned}
C_{x}(t, f, c) & =\int_{\tau=-\infty}^{+\infty} A \phi_{T}(\tau) e^{j 2 \pi\left(f_{0}(t+\tau)+\frac{c_{0}}{2}(t+\tau)^{2}\right)} \ldots \\
& =\int_{\tau=-\infty}^{+\infty} A \phi_{T}(\tau) e^{j 2 \pi\left(\tau^{2}\left(c_{0} / 2-c / 2\right)\right)} \ldots \\
& =\int_{\tau=-\infty}^{+\infty} A \phi_{T}(\tau) e^{j \psi(\tau)} d \tau
\end{aligned}
$$

We consider here cases where $c_{0}-c \neq 0$, the case $c_{0}=c$ leading to the classical Fourier transform.

The integrals of the form (9) can be approximated by the stationary phase approximation [10] if $A \phi_{T}(\tau)>0$ and $\psi(\tau)$ are both $C^{1}$, and if $A \phi_{T}(\tau)$ varies slowly compared to the oscillations controlled by the phase $\psi(\tau)$. In this case, negative and positive values of $e^{j \psi(\tau)}$ tend to cancel each other, except near points where the phase is stationary. To determine these points, we search for the time $\tau_{0}$ such that the derivative of the phase is zero:

$$
\begin{aligned}
\dot{\psi}\left(\tau_{0}\right) & =0 \\
2 \pi\left(\tau_{0}\left(c_{0}-c\right)+f_{0}-f+c_{0} t\right) & =0 \\
\tau_{0} & =\frac{f-f_{0}-c_{0} t}{c_{0}-c}
\end{aligned}
$$

Given that $\ddot{\psi}\left(\tau_{0}\right)=2 \pi\left(c_{0}-c\right)$, this stationary point is nondegenerate. In the general case, the phase can be approximated by a Taylor expansion. In the case of linear chirps (1), the Taylor expansion leads to the exact result

$$
\psi(\tau)=\psi\left(\tau_{0}\right)+\left(\tau-\tau_{0}\right)^{2} \frac{\ddot{\psi}\left(\tau_{0}\right)}{2}
$$

More generally, the stationary phase approximates equation (9) by

$$
C_{x}(t, f, c) \approx A \phi_{T}\left(\tau_{0}\right) e^{j \tau_{0}} \int_{\tau=-\infty}^{+\infty} e^{j\left(\tau-\tau_{0}\right)^{2} \frac{\ddot{\psi}\left(\tau_{0}\right)}{2}} d \tau .
$$

With the change of variables

$$
\begin{aligned}
& u^{2}=\left(\tau-\tau_{0}\right)^{2} \frac{\ddot{\psi}\left(\tau_{0}\right)}{2}, \\
& d \tau=\sqrt{\frac{2}{\ddot{\psi}\left(\tau_{0}\right)} d u,}
\end{aligned}
$$

approximation (14) becomes

$$
C_{x}(t, f, c) \approx A \phi_{T}\left(\tau_{0}\right) e^{j \psi\left(\tau_{0}\right)} \sqrt{\frac{2}{\ddot{\psi}\left(\tau_{0}\right)}} \int_{u=-\infty}^{+\infty} e^{j u^{2}} d u .
$$

Using the Fresnel integral

$$
\int_{-\infty}^{+\infty} e^{j u^{2}} d u=\sqrt{\frac{\pi}{2}}+j \sqrt{\frac{\pi}{2}},
$$

the chirplet transform is approximated by

$$
C_{x}(t, f, c) \approx A \phi_{T}\left(\tau_{0}\right) e^{j \psi\left(\tau_{0}\right)} \sqrt{\frac{2}{\ddot{\psi}\left(\tau_{0}\right)}}(1+j) \sqrt{\frac{\pi}{2}}
$$

Inserting the values of $\tau_{0}$ and $\ddot{\psi}\left(\tau_{0}\right)$ leads to

$$
\begin{aligned}
C_{x}(t, f, c) & \approx A \sqrt{\frac{1}{c_{0}-c}} \phi_{T}\left(\frac{f-\left(c_{0} t+f_{0}\right)}{c_{0}-c}\right) e^{c^{\prime} f^{2}+f^{\prime} f+\Phi^{\prime}}, \\
c^{\prime} & =\frac{-\pi}{c_{0}-c}, \\
f^{\prime} & =\frac{2 \pi}{c_{0}-c}\left(f_{0}+c_{0} t\right), \\
\Phi^{\prime} & =\frac{-\pi}{c_{0}-c}\left(f_{0}+c_{0} t\right)^{2}+f_{0} t+\frac{c_{0}}{t^{2}}+\frac{\pi}{4}
\end{aligned}
$$


The approximation holds for positive and smooth analysis windows $\phi_{T}(\tau)$. In this case, the phase of the chirplet transform of a windowed chirp (20) has the same behaviour as a time chirp. At a given time $t$, the spectrum has the shape of the analysis window $\phi_{T}(\tau)$ dilated by a scaling factor $c_{0}-c$ and centred on the frequency $f_{0}+c_{0} t$. For symmetric window, it can be rewritten:

$$
\phi_{T}\left(\frac{c_{0} t+f_{0}-f}{c_{0}-c}\right)=\phi_{\left|c_{0}-c\right| T}\left(f-\left(c_{0} t+f_{0}\right)\right)
$$$$
\sqrt{(24) \frac{1}{\left|d_{0}-d\right|}} \phi_{\left|d_{0}-d\right| M K}\left[k-\left(k_{0}+d_{0} n\right) K\right]
$$

green curves are the values approximated by (28) and (27) respectively, the red curve is the value of $|C[0,0, d]|$.

The transition from approximation (27) to (28) corresponds to the value of $\left|d_{0}-d\right|$ such that these two approximations are equal.

$$
A\left|\Phi_{M}\left[k-\left(k_{0}+d_{0} n\right) K\right]\right|=
$$

The specific case of the rectangular windows leads to an exact result based on Fresnel integrals, which can be approximated in the same way as smooth windows [11].

\section{B. Discrete chirplet transform}

With a sampling step $\delta_{t}$, the discretization of signal (6) is

$$
x[m]=x\left(t=m \delta_{t}\right)=A e^{j 2 \pi\left(k_{0} m+\frac{d_{0}}{2} m^{2}\right)}
$$

where $k_{0}=f_{0} \delta_{t}$ and $d_{0}=c_{0} \delta_{t}^{2}$ are the reduced frequency and chirprate respectively.

The discrete version of the chirplet transform (3) is

$C[n, k, d]=\sum_{m=-M}^{+M} x[n+m] \phi_{M}[m] e^{-j 2 \pi \frac{1}{2} \frac{l}{L} d_{\max } m^{2}} e^{-j 2 \pi m \frac{k}{K}}$,

with $n, k$ are the time and frequency indices respectively, $K$ the number of frequencies. The chirprate is $d=\frac{l}{L} d_{\max }$, with a chirprate index $l$ ranging from from $-L$ to $+L$, leading to $2 L+1$ different chirprates. The discrete smoothing window $\phi_{M}[m]$ has $2 M+1$ points. Note that contrary to $k$ which is the frequency bin index, the signal frequency $k_{0}$ is a reduced frequency. The corresponding frequency index $k_{0} K$ is not bound to be an integer. Compared to the continuous case, two parameters have to be chosen for the chirprate discretization: the maximal chirprate $d_{\max }$, which can be chosen a priori of the analysed signal, and the chirprate step $\frac{2 d_{\max }}{2 L+1}$.

To simplify the notations, we only consider in the following the norm of $C[n, k, d]$.

The discretization of the (20) leads to

$$
|C[n, k, d]| \approx A \sqrt{\frac{1}{\left|d_{0}-d\right|}} \phi_{\left|d_{0}-d\right| M K}\left[k-\left(k_{0}+d_{0} n\right) K\right]
$$

However, for very small $\left|d_{0}-d\right|$, the chirplet transform may be better approximated by the Fourier transform of a wave:

$$
|C[n, k, d]| \approx A\left|\Phi_{M}\left[k-\left(k_{0}+d_{0} n\right) K\right]\right|
$$

where $\Phi_{M}[k]$ is the discrete Fourier transform of $\phi_{M}[m]$. The expression $\left(k_{0}+d_{0} n\right) K$ is a non-integer frequency index corresponding to the central frequency of the signal $x[\mathrm{~m}]$ windowed at time $n$. Note that the maximum of $\Phi_{M}[k]$ is $\Phi_{M}[0]=\sum \phi_{M}[m]$.

Figure 1 illustrates the accuracy of the Fourier and stationary phase approximations. We consider the special case $k_{0}=d_{0}=0$ and $A=1$, and look at the maximal value of $|C[n, k, d]|=|C[0,0, d]|$ for $M=1000$. The blue and

$$
\left|d_{0}-d\right| \quad=\frac{1}{\Phi_{M}[0]^{2}}=\Delta_{d 0}
$$

Consequently, the spectrum of the chirp signal (25) will be approximated by:

$$
|C[n, k, d]| \approx\left\{\begin{array}{l}
A\left|\Phi_{M}\left[k-\left(k_{0}+d_{0} n\right) K\right]\right| \\
\quad \text { if }\left|d_{0}-d\right|<\frac{1}{\Phi_{M}[0]^{2}}=\frac{1}{\left(\sum_{m} \phi_{M}[m]\right)^{2}} \\
A \sqrt{\frac{1}{\left|d_{0}-d\right|}} \phi_{\left|d_{0}-d\right| M K}\left[k-\left(k_{0}+d_{0} n\right) K\right] \\
\quad \text { otherwise. }
\end{array}\right.
$$

The energy of the chirplet coefficient is maximal if $\mid d_{0}-$ $d \mid<\frac{1}{\left(\sum_{m} \phi_{M}[m]\right)^{2}}$. Consequently, by choosing the parameters of the chirplet transform are such that chirprate step $\Delta_{d}=\frac{d_{\max }}{L}$ is less than $2 \Delta_{d 0}=\frac{2}{\left(\sum_{m} \phi_{M}[m]\right)^{2}}$, we are assured to get at least a chirplet coefficient corresponding to the first approximation, and consequently with the highest possible energy, for any chirp signal.

This chirplet step depends on the energy of the analysing window $\phi_{M}[m]$, which is non-normalized: its maximum value is 1. Longer windows have higher energy, and thus need a smaller chirplet step to achieve good detection. This result confirms the intuitive idea that long windows are able to distinguish smaller differences of chirprates than short windows.

\section{MAXIMUM CHIRPLET TRANSFORM}

To simplify the detection problem, we define the Maximum Chirplet Transform (MCT) $D[n, k]$ at a given time-frequency point $[n, k]$, containing all maxima of the square modulus of the chirplet coefficients along the chirprates

$$
D[n, k]=\max _{d}|C[n, k, d]|^{2} .
$$

Our aim is to use the MCT to provide a representation through which we can detect chirp-like signals. Direct detection in the MCT leads to a wide spectral window, as detailed below. To mitigate this problem we propose selecting significant coefficients through an iterative selection strategy, reminiscent of MP.

A similar approach is proposed in [12], called peak-picked Chirp Fourier transform (ppCFT). At each time $n$, the value of the chirprate leading to the maximum peak is identified, and the associated coefficients are selected to form the ppCFT at time $n$. This approach leads to narrow spectral window, equal to the spectral window of a stationary wave, but can handle only a single chirp. 

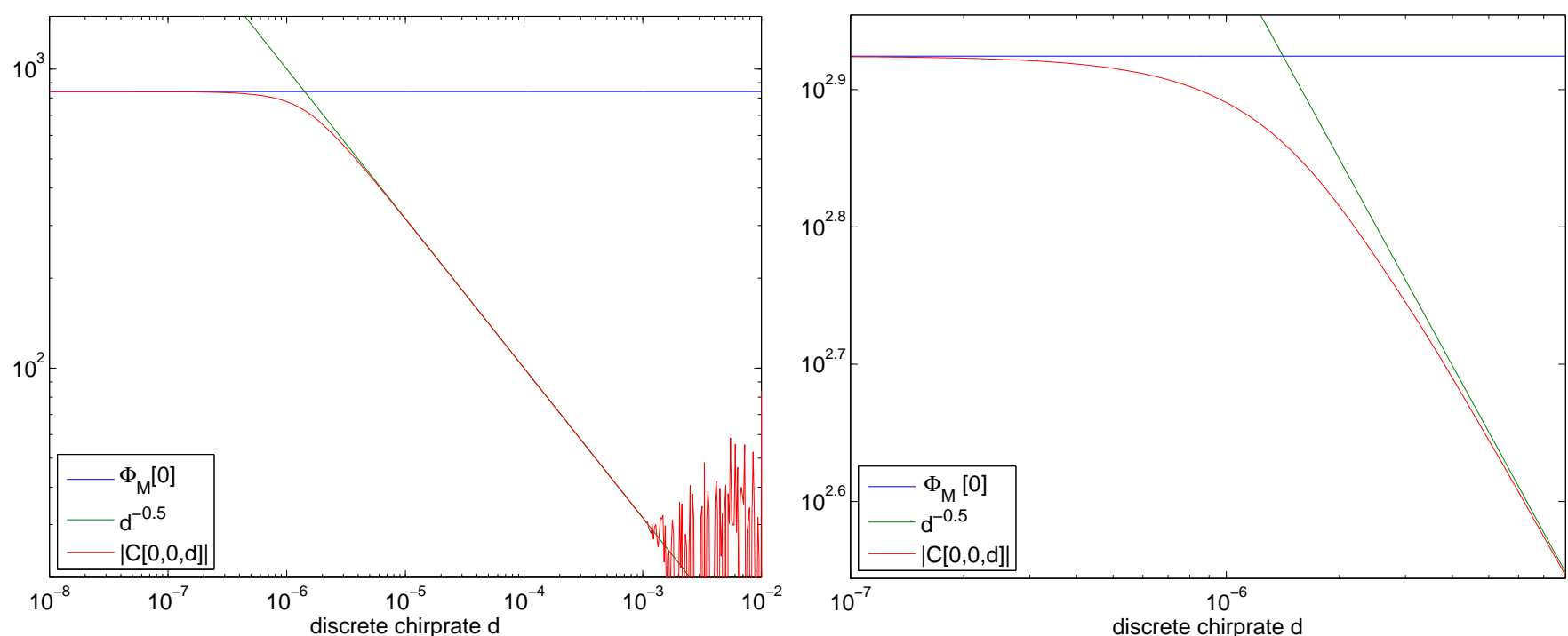

Fig. 1. Blue: approximated value of $|C[0,0, d]|$ with approximation (28), valid for small values of $\left|d-d_{0}\right|$. Green: approximated value of $|C[0,0, d]|$ with approximation (27), valid for large values of $\left|d-d_{0}\right|$. Red: computed value of $|C[0,0, d]|$. The first approximation is valid up to $d=\frac{1}{\Phi_{M}[0]^{2}}$, while the second is valid after. For too large values of $c$ such that $c T>\frac{1}{\delta_{t}}$ a spectral aliasing appears. The right figure is a zoom of the left figure near the transition between the two approximations.

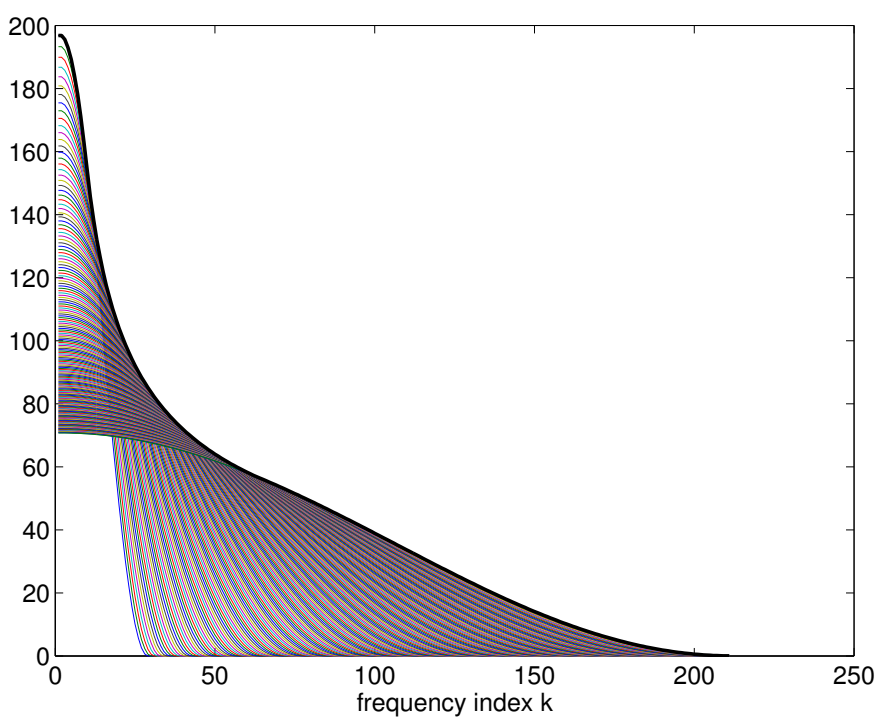

Fig. 2. Illustration of the determination of the upper-bound spectral window $\Phi_{u}[k]$ (35). The set of spectral windows coming from different values of the chirprate parameter of the chirplet transform is plotted in different colours. At each frequency bin, the upper-bound spectral window correspond to the highest point, plotted in bold black.

\section{A. Spectral window of the MCT}

The spectral window of the MCT, denoted $\Phi_{M C T}(f)$ in the continuous case, is the magnitude spectrum corresponding to a single chirplet. It is defined by using approximation (31)

$$
\left.\Phi_{M C T}(f)=\max \left\{\left|\Phi_{T}(f)\right|, \max _{c}\left\{\mid c^{-1 / 2} \phi_{T}(f / c)\right) \mid\right\}\right\}
$$

At a given $f$, the second member of the parenthesis is found by solving $\left.\frac{d}{d c} \mid c^{-1 / 2} \phi_{T}(f / c)\right) \mid=0$. Unfortunately, this equation does not have a closed form solution in the general case.

In the discrete case, frequency and chirprate are discrete, and the chirprates are bounded by $d_{\max }$. The spectral window is consequently easily computable. However, in the general case the spectral window at discrete time $n$ is centred on the frequency-chirprate location $\left[k_{0}+d_{0} n, d_{0}\right]$ whose values are not bound to the discrete values of the chirplet transform. We will consider instead an upper-bound spectral window, denoted $\Phi_{u}[k]$, centred on the frequency 0 , such that for any MCT magnitude spectrum, the upper-bound spectral window normalized and centred on the highest MCT value is greater or equal to the MCT spectrum generated from an associated chirplet at the time and frequency.

The size of the widest window is determined when the value of the analysed signal's chirprate $d_{0}$ is equal to $d_{0}= \pm d_{\max }$. 

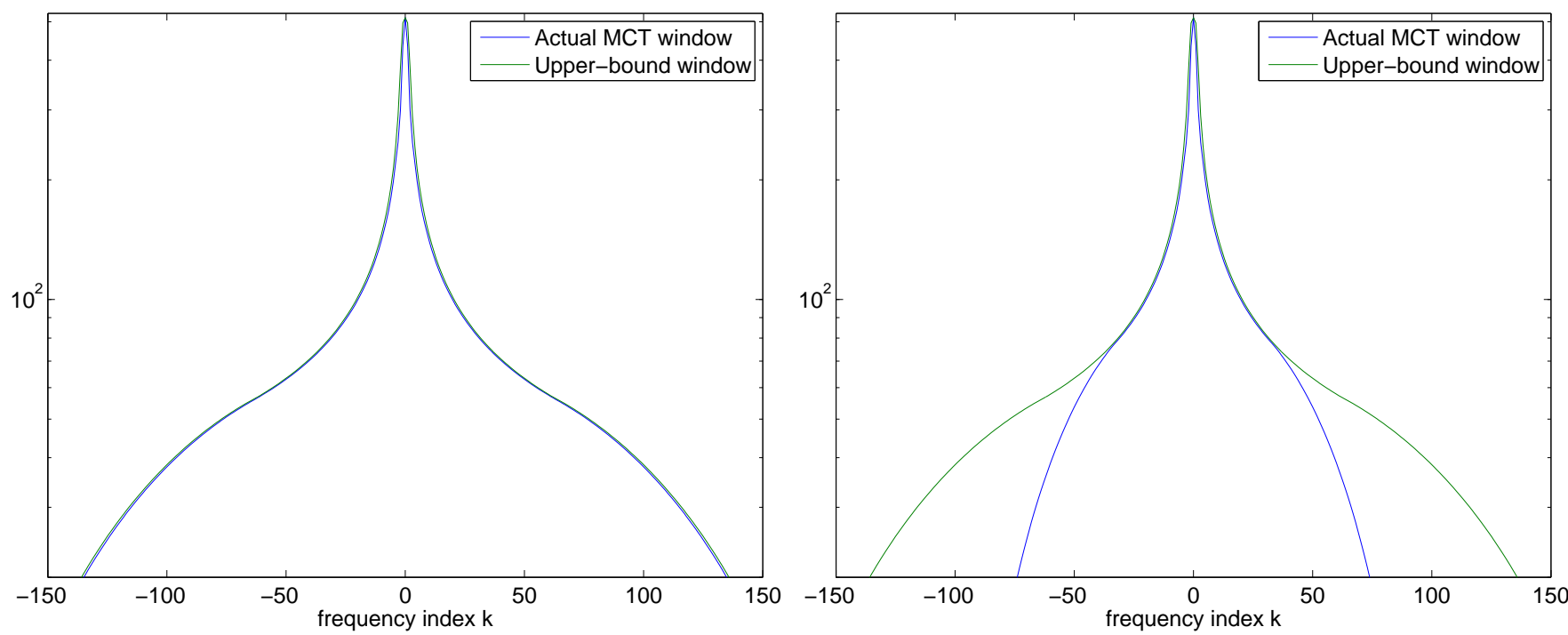

Fig. 3. Comparison of the upper-bound spectral window with two different MCT spectral windows, for a small chirprate step $\Delta_{d}$. In green, the upper-bound spectral window, computed for a Hanning window of 1023 points, 2048 frequency bins and 51 different chirprates from $-10^{-4}$ to $+10^{-4}$. Left in blue, the MCT spectrum of a chirp with chirprate equal to the maximum chirprate $10^{-4}$. The MCT spectrum is close to the upper-bound spectral window, but always less than or equal to. Right in blue, the MCT spectrum of a pure wave (chirprate zero). The MCT spectrum is narrower than in the previous case.

For such a signal, the most distant chirplets getting some signal energy, whose chirprates are $d=-d_{0}$, are as far as $k_{0} \pm \mid d-$ $d_{0} \mid \frac{M-1}{2}=k_{0} \pm d_{\max } M$. This value gives the half-length of the spectral window $\Phi_{u}[k]$. Consequently, when considering a generic signal arbitrarily set to $k_{0}=d_{0}=0$, the maximum analysing chirprate is $2 d_{\max }$.

First, we assume that the chirprate step is small enough to get at least one chirplet coefficient in the first approximation of (31). In this case, the upper-bound spectral window $\Phi_{u}[k]$ is approximated by taking at each frequency $k$ the maximum between the spectral window $\left|\Phi_{M}[k]\right|$ and the set of $D_{i}$ chirped spectral windows $\sqrt{\frac{1}{\left|d_{i}\right|}} \phi_{\left|d_{i}\right| M K}[k]$ whose sizes are integer values.

$$
\begin{gathered}
\Phi_{u}[k]=\max \left(\Phi_{M}[k], \max _{d_{i}} \sqrt{\frac{1}{\left|d_{i}\right|}} \phi_{\left|d_{i}\right| M K}[k]\right), \\
\frac{1}{\Phi_{M}[0]^{2}} \leq d_{i} \leq 2 d_{\text {max }}, \text { such that }\left|d_{i}\right| M K \in \mathbb{N}
\end{gathered}
$$

Note that the highest value of a local maxima of a spectrum is achieved when the discrete frequency of a wave corresponds exactly to a frequency bin, that is $k_{0}=\frac{n_{1}}{K}$, with $n_{1}$ an integer. On the other hand, the highest values for the points near this local maxima, that is points belonging to the lobes of the spectral window, are achieved when the discrete frequency of a wave is $k_{0}=\frac{n_{1}+0.5}{K}$. The closest point to the maximum will have approximately the same value. Consequently, odd window sizes, that are windows centred on a frequency bin, never give maximum values on a frequency different from the window centre and thus can be discarded from the computation.

Figure 2 illustrates the design of this spectral window, limited on the right part of this window. On each frequency bin, the highest value over all possible windows is selected to make the upper-bound spectral window.

Figure 3 compares the upper-bound spectral window with the actual MCT window in two cases of chirplet transform. In these two cases, the upper-bound spectral window is indeed higher that the MCT spectral window.

In the undesirable case of a large chirprate step $\Delta_{d}>2 \Delta_{d 0}$, the maximal energy of the chirplets depends on the signal's chirprate. In the worst case, the signal's chirprate is halfway between two discrete chirprates of the chirplet transform, leading to smaller but wider spectral window, corresponding to a value of $\left|d_{0}-d\right|=\Delta_{d} / 2$ in approximation (31). The upper-bound spectral window is computed following equation (35), but with chirprate $d_{i}$ such that $\Delta_{d} / 2 \leq d_{i} \leq 2 d_{\max }$.

As shown on figure 4, the upper-bound spectral window is much wider and decreases much more slowly than the upperbound spectral window coming from the small chirprate step. Consequently, this window is a bad approximation when the signal's chirprate is close to a discrete chirprate of the chirplet transform. This illustrates the need to select $\Delta_{d} \leq 2 \Delta_{d 0}$.

\section{B. Detection in the MCT}

The goal of this section is to select a set of significant timefrequency coefficients in order to enable subsequent estimation of the signal's chirps. Additionally, we try to select as few coefficients as possible, to limit the computation needed for the chirp estimation presented in the next section.

A first step in the selection of chirplet coefficients is to determine whether a time-frequency coefficient contains significant signal energy, called hypothesis $\mathcal{H}_{1}$, or not, the hypothesis $\mathcal{H}_{0}$.

The Maximum Chirplet transform coefficients (32) are chosen among squared modulus chirplet coefficients, representing the coefficients' energy. A chirplet correlated with the signal should lead to a chirplet coefficient of higher energy than chirplet coefficients containing noise only, and so should the MCT coefficient. In other words, the hypothesis test for a 

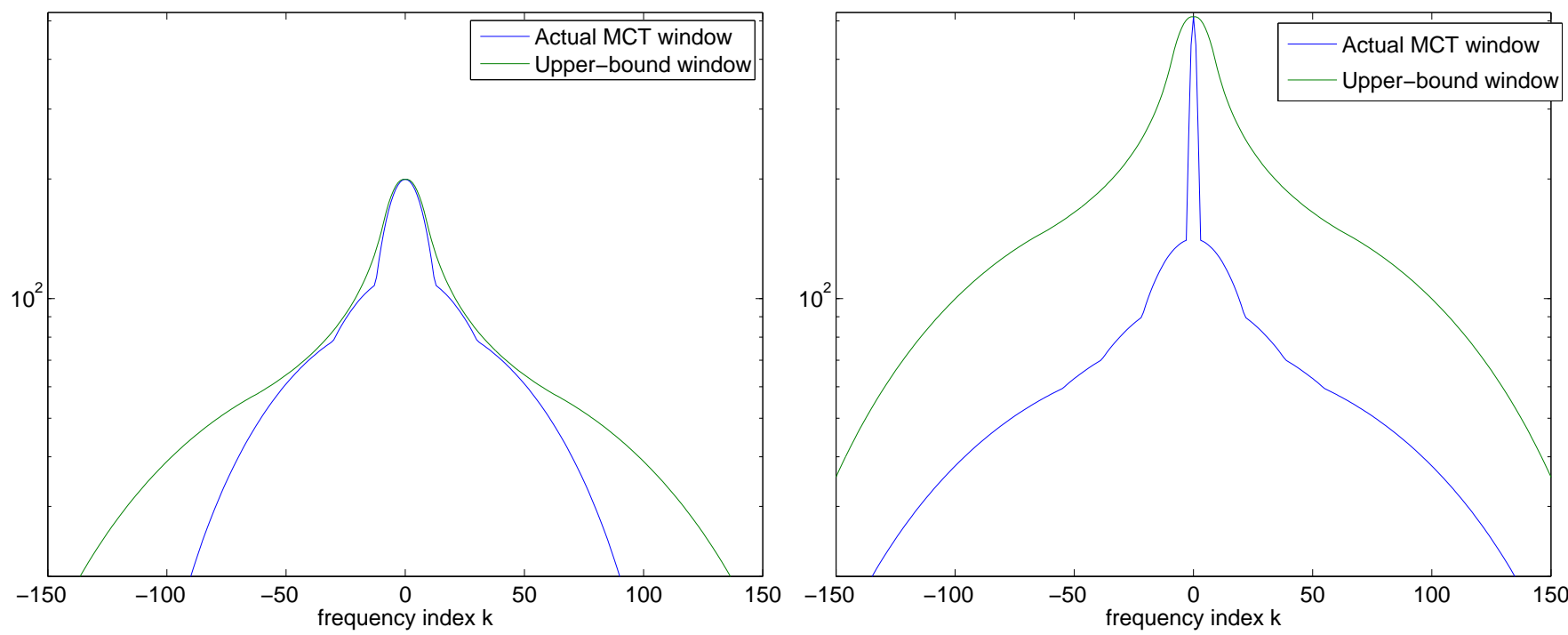

Fig. 4. Comparison of the upper-bound spectral window with two different MCT spectral windows, for a high chirprate step $\Delta_{d}$. In green, the upper-bound spectral window, computed for a Hanning window of 1023 points, 2048 frequency bins and 5 different chirprates from $-10^{-4}$ to $+10^{-4}$. Left in blue, the MCT spectrum has a chirprate of $1.25 \times 10^{-5}$, leading to a very poor MCT spectrum. However, the peak of the upper-bound spectral window follows correctly the MCT spectrum. Right in blue, the MCT spectrum of a chirp with chirprate equal to $5 \times 10^{-5}$, corresponding to a chirprate of the chirplet transform. When adjusting the upper-bound spectral window to the maximal value of the MCT spectrum, the upper-bound spectral window has a very large peak and a very high tail, leading to poor frequency and poor resolutions.

single chirplet is solved by selecting an appropriate detection threshold $t$. If the MCT coefficient is below this threshold it is assumed to contain noise energy only.

In the hypothesis $\mathcal{H}_{0}$, the chirplet coefficient contains Gaussian noise of variance $\sigma^{2}$ only, and consequently has a circular complex Gaussian distribution, and its square modulus has a $\chi^{2}$ distribution with two degrees of freedom. Consequently, $D[n, k]$ is the maximum of $2 L+1$ random variables having a $\chi^{2}$ distribution with two degrees of freedom.

We assume here that at a given time-frequency location $[n, k]$, all chirplet coefficients $C[n, k, d]$ are independent. Under this assumption, which is studied in next section, the order statistics [13] leads to the distribution of $D[n, k]$ for noise only of $f_{D[n, k], \mathcal{H}_{0}}$

$$
f_{D[n, k], \mathcal{H}_{0}}(x)=(2 L+1)\left(1-e^{-\frac{-x}{2 \sigma^{2}}}\right)^{2 L} \frac{1}{2 \sigma^{2}} e^{-\frac{-x}{2 \sigma^{2}}}
$$

In this paper, the detection strategy chosen is a NeymanPearson approach. With a chosen probability of false alarm $p_{f a}$, the threshold $t$ is such that

$$
\begin{aligned}
& t \quad / \mathcal{P}\left(D[n, k]_{\mathcal{H}_{0}}>t\right)=p_{f a} \\
& \quad t=-\sigma^{2} \ln \left(1-\left(1-p_{f a}\right)^{\frac{1}{2 L+1}}\right)
\end{aligned}
$$

This threshold depends on the noise level $\sigma^{2}$, which must be estimated. To do this, we can use a noise level estimation based on the spectral kurtosis of the minimal statistics [14]. This estimation is not discussed in this paper, and we consider in the following that the noise level is known. In order to avoid a large number of false detections we choose a low probability of false alarm, e.g. $p_{f a}=10^{-5}$.

At this stage we might be tempted to perform detection by a direct thresholding of all the MCT coefficients. This would lead to a set of detected points $\mathcal{S}=\{[n, k]: D[n, k]>t\}$.
However, due to the large width of the MCT spectral window, this will result in a large number of additional false alarms in the neighborhood of actual significant coefficients, particularly in high signal-to-noise (SNR) scenarios. To avoid this phenomenon we propose a computationally efficient interference cancellation strategy, detailed next.

\section{Iterative masking}

To avoid this over-selection of coefficients, we introduce an iterative masking approach that can be viewed as a conservative and computationally efficient variant of MP. At each iteration, the highest MCT spectrum coefficient is selected. The upper-bound spectral window (35) centred on this point is subtracted from the MCT spectrum, thereby guaranteeing to remove the energy associated with a detected chirplet at that time frequency point. A new iteration is then run over the residual spectrum. The iterations stop when all spectrum coefficients are below the detection threshold $t$. Contrary to the Matching Pursuit approach, the spectrum does not need to be re-computed at each iteration.

Figure 5 shows the first three iterations of such a detection scheme on a test signal. Only a single coefficient is selected for each chirp, without an important loss of frequency resolution. Note that interferences between close chirps may lead to values higher than the upper-bound spectral window of the highest chirp. These interferences are associated with neighboring chirps and are subsequently reduced through the subtraction of the upper bound spectral window for the second peak.

The use of this iterative masking leads to a loss of detection range, that depends on the chirprate of the detected chirplet. When the signal's chirprate is equal to the maximal chirprate of the chirplet transform, the MCT spectral window is identical 

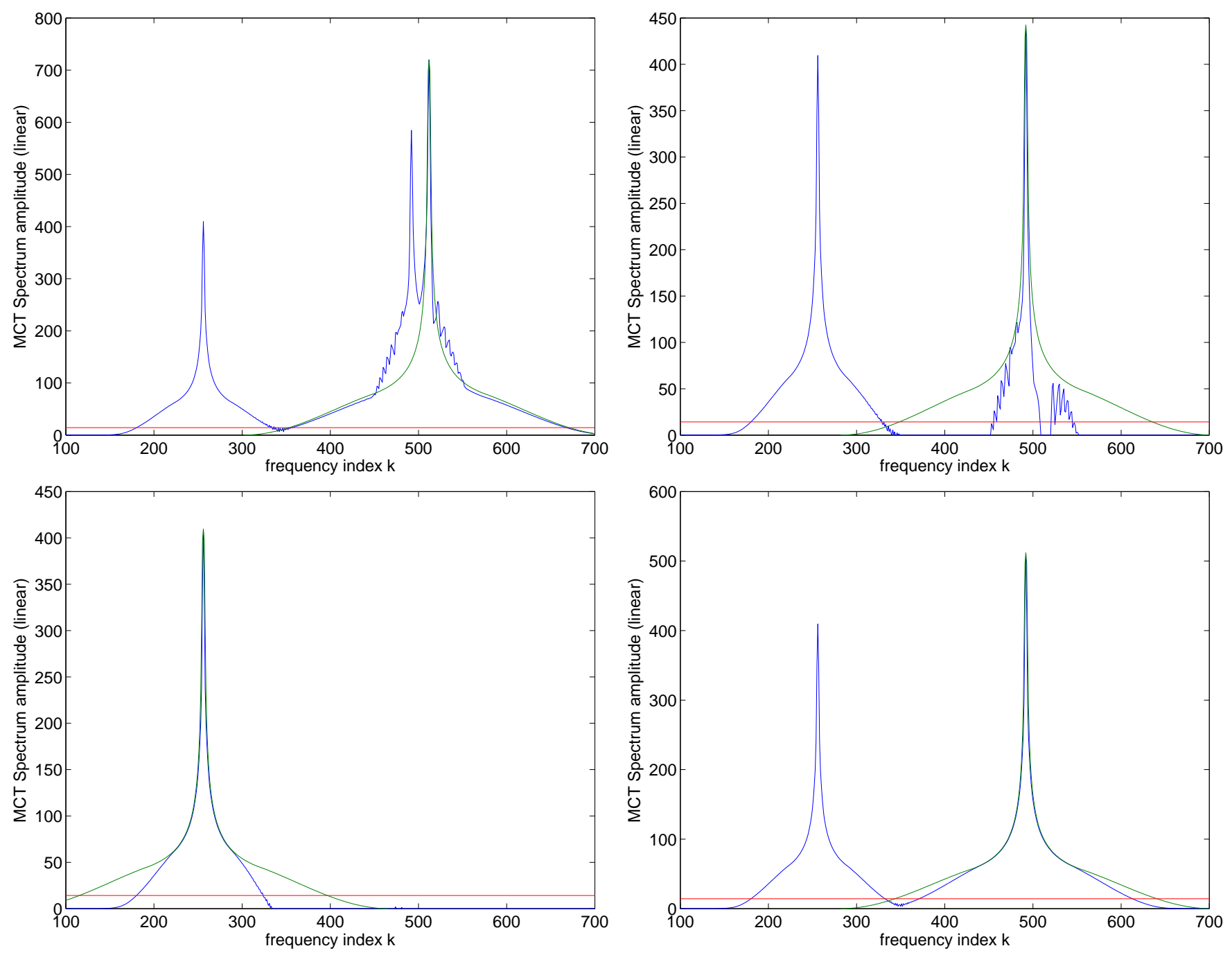

Fig. 5. Illustration of the iteration detection algorithm. Up left, MCT spectrum of a test example with a Gaussian noise of variance $\sigma^{2}=1$. In green the upper-bound spectral window centred on the highest points of the spectrum. In red the detection threshold $t$. Up right, the MCT spectrum at the second iteration. The negative values are set to zero for a better visualisation. The third iteration is given on bottom left, the negative values are again set to zero. At each iteration, the MCT spectrum can be understood as an approximation of the MCT spectrum of the remaining chirps only. As a comparison with the approximation at the second iteration (up left), the MCT spectrum of the two last chirps only is given on bottom right.

to the upper-bound spectral window (cf Fig. 3, left). Consequently, the spectral subtraction does not remove inappropriate spectral energy. On the other hand, in the worst case the signal's chirprate is 0 , and the MCT spectral window is narrower than the upper-bound spectral window. More spectral energy than required is removed, which will lead to a loss of detection. The worst case detection loss is quantified by the difference between the two curves on the right hand side of Fig. 3 (we will always choose a small enough chirprate step to avoid the case illustrated in Fig. 4). For clarity the difference is plotted on $\mathrm{dB}$ scale in Fig. 6. The worst case detection loss has two main regimes. The main lobe can be considered as a resolution loss that will suppress the selection of other chirplets that are close in frequency. The maximum loss here is $-8 \mathrm{~dB}$ and has a width of 5 frequency bins. In the side lobes the detection loss is significantly lower reaching a maximum of $-11 \mathrm{~dB}$. Thus within these limits we can expect the iterative masking to provide robust detection.

\section{Correlation in the MCT}

This section investigates the correlation between chirplet coefficients $C[n, k, d]$ at a given time-frequency location $[n, k]$, and the validity of the distribution of $D[n, k]$ for noise only (36). To do this, we consider the Kolmogorov-Smirnov test [15], which is a non parametric test comparing a sample with a distribution of reference. Given the empirical distribution $F_{n}(x)$ of $n$ observations $X_{j}$, the Kolmogorov-Smirnov statistic $K S_{n}(x)$ is defined as

$$
K S_{n}=\sup _{n}\left|F_{n}(x)-F(x)\right|,
$$

with $F(x)$ the cumulative distribution function of reference.

The distribution of Kolmogorov $K$ is

$$
K=\sup _{t \in[0,1]}|B(t)|
$$

with $B(t)$ the Brownian bridge. Its cumulative distribution 


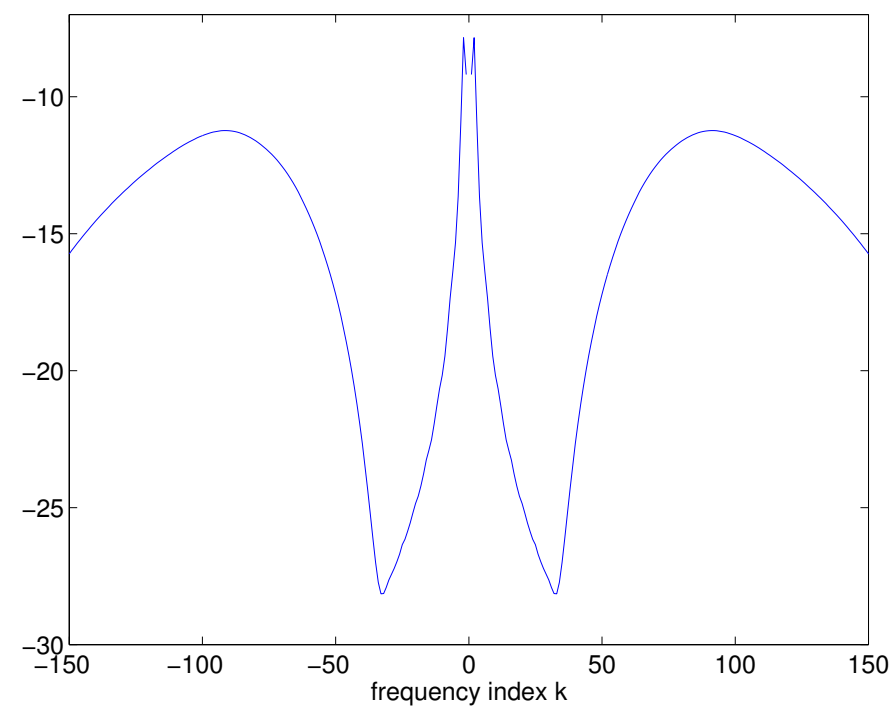

Fig. 6. Illustration of the detection loss in the worst case scenario, illustrated on Figure 3, right. The detection loss is the difference between the upper-bound spectral window and the actual MCT spectrum of the signal. Amplitude is normalized by the maximum of the MCT spectrum.
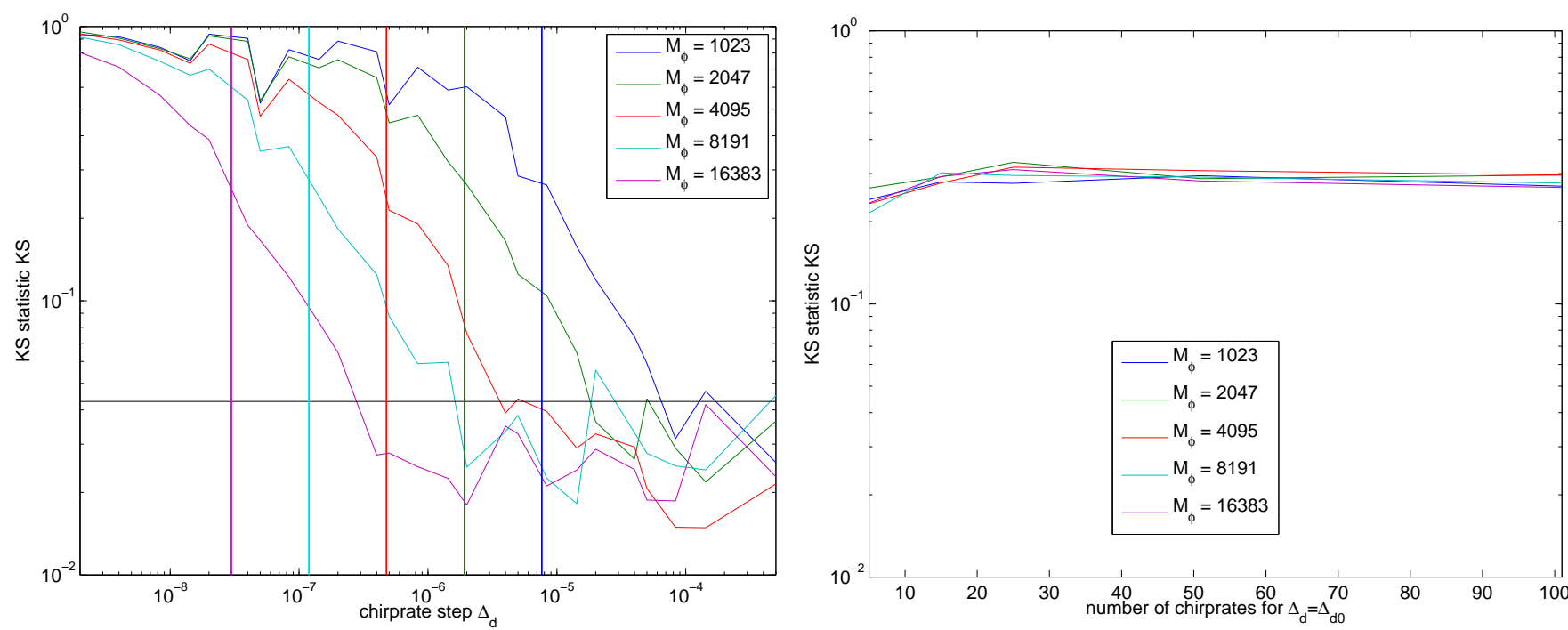

Fig. 7. Left, values of the Kolmogorov-Smirnov Statistic comparing the distribution of the maximum chirplet transform at a given time-frequency location $[n, k]$ and the distribution coming from independent coefficients (36), for different parameters. The black line shows the level for which the null hypothesis is rejected. At a given window's size, there is a threshold value of the chirprate step $\Delta_{d}=\frac{d_{\max }}{L}$ leading to a good approximation of the maximum chirplet distribution. The vertical lines correspond to the maximal chirprate step $2 \Delta d_{0}$ (30) assuring good detection. Right, values of the Kolmogorov-Smirnov Statistic for $\Delta_{d}=2 \Delta d_{0}$, for different number of chirprates. This statistic is independent of the number of chirprates for a given chirprate step.

function is

$$
\mathcal{P}(K \leq \alpha)=1-2 \sum_{i=1}^{\infty}(-1)^{i-1} e^{-2 i^{2} \alpha^{2}}
$$

Under the null hypothesis, the Kolmogorov-Smirnov statistics converges such that

$$
\sqrt{n} K S_{n} \underset{n \rightarrow \infty}{\longrightarrow} \sup _{t}|B(F(t))|
$$

The Kolmogorov-Smirnov test rejects the null hypothesis with a probability of false alarm $\alpha$ if

$$
\sqrt{n} K S_{n}>K_{\alpha}, \text { with } \mathcal{P}\left(K \leq K_{\alpha}\right)=1-\alpha
$$

In practice, the convergence (42) is a good approximation for $n>100$, and $K_{\alpha}$ is approximated by 1.358 for $\alpha=5 \%$.
In our case, we want to check if the distribution of the Maximum Chirplet coefficient $D[n, k]$ is the same as the one coming from uncorrelated chirplet coefficients (36), which is the null hypothesis, or not. The cumulative distribution function $F(x)$ derives from (36)

$$
F(x)=\left(1-e^{-\frac{-x}{2 \sigma^{2}}}\right)^{(2 L+1)}
$$

This distribution is compared with the distribution of the $D[n, k]$ at $n=1$ and at the normalised frequency 0.25 , computed over 1000 realisations of noise, for different size of windows $2 M+1$, different maximum chirprates $d_{\max }$ and different number of chirplets $2 L+1$. The key parameters are the window's length and the chirprate step, defined as $\Delta_{d}=\frac{d_{\max }}{L}$. The KS statistics $K S_{n}$ are shown on figure 7 


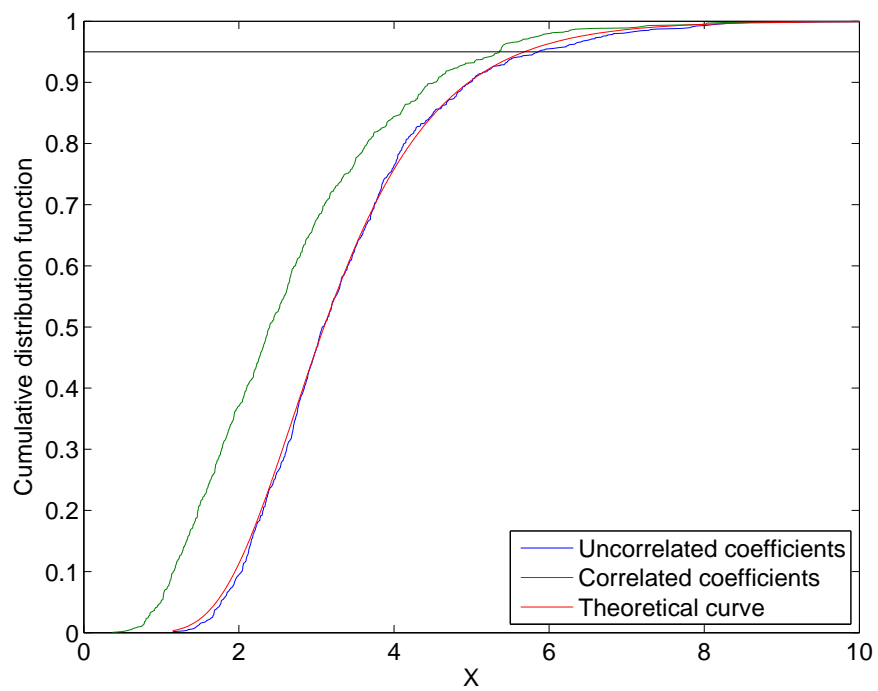

Fig. 8. Influence of the correlation between chirplet coefficients on the cumulative distribution function of the maximum chirplet. The empirical cumulative distribution functions are plotted for 1000 realisations of noise. The correlated coefficients comes from a chirplet transform with a window's length of 4095 , 15 chirprates and a chirprate step of $\Delta_{d 0}$. The uncorrelated coefficients are sets of 15 independent Gaussian noise realisations.

for different values of $M$. When the chirprate step increases, the KS statistic decreases down to a value where the null hypothesis is not rejected anymore. The step size at which the null hypothesis is no longer rejected is smaller for longer windows.

Figure 8 shows the influence of correlations on the cumulative distribution function of the maximum chirplet coefficient. In presence of correlation between the chirplet coefficients at a given time-frequency location, the cumulative distribution function increases faster than without correlation. The black line indicates the value $F(x)=0.95$. Its intersection between the green and blue curves indicates a detection threshold corresponding to a probability of false alarm of $5 \%$. With correlated coefficients, this threshold is lower. In other words, the detection threshold described in section III-B leads to a higher probability of false alarm than expected when chirplets coefficients are correlated.

In conclusion, the distribution (36) is a good approximation of the maximum chirplet coefficient distribution in the case of long windows associated with high enough chirprate step. In case of correlation, the detection threshold of section III-B leads to a higher probability of false alarm than expected.

\section{Algorithm}

The previous section detects a set of maximum chirplet coefficients, associated with their corresponding chirprate. The next goal is to gather these chirplets into different signals, while limiting the computational cost, i.e. limiting the chirplet transform redundancy.

\section{A. Gathering into chirps}

First, we aim to gather the set of detected chirplets into a small set of chirps in a real time framework, these chirps corresponding to each linear part of the analysed signal modelled by (2). Each chirp is characterised by four parameters:

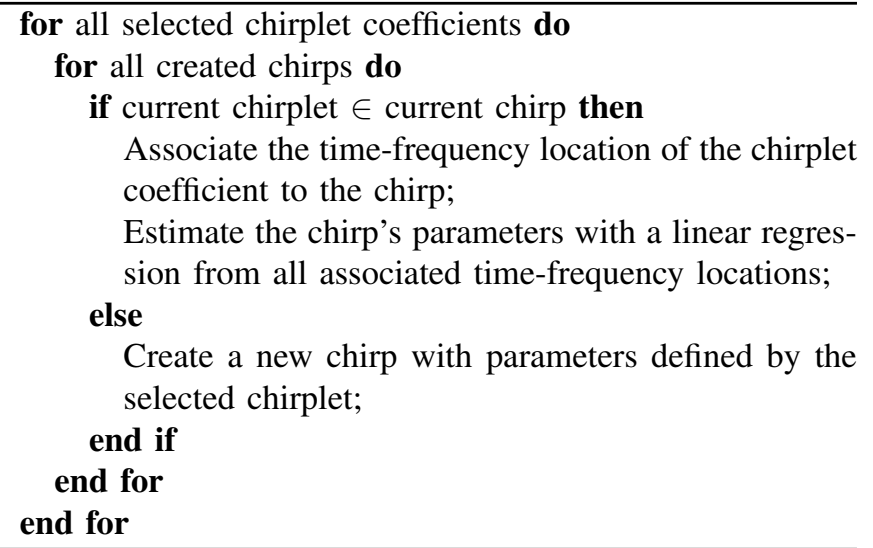

the starting point $n_{s}$, the end point $n_{e}$, the initial frequency $k_{i}$ at time $n_{s}$ and the chirprate $d_{r}$.

For all selected chirplet coefficients, from the smallest time index and frequency index to the highest indices, we check if the chirplet coefficients fit in an existing chirp, and update that chirp's parameters with the new coefficient. Otherwise, we create a new chirp, whose starting point and end point are the first and last points of the window associated with the chirplet coefficient. The chirprate is set to the chirplet's chirprate, and the initial frequency is deduced from the central frequency of the chirplet and its chirprate. The algorithm is summarised as follows: The conditions for associating a chirplet coefficient, centred on time index $n$ and frequency index $k$ with a chirprate $d$, to a chirp are:

* time condition, the chirp and the chirplet overlap:

$$
n_{s} \leq n \leq n_{e}+\delta_{n}
$$

* chirprate condition, the chirplet and the chirps have approximately the same chirprate:

$$
\left|d-d_{r}\right| \leq \delta_{d}
$$


* frequency condition, the central frequency of the chirplet is located on the chirp:

$$
\left|k_{i}+d_{r}\left(n-n_{i}\right)-k\right| \leq \delta_{k}
$$

Each selected chirplet is associated to only one chirp. In other words, chirplets located at the crossing of two chirps can be linked to only one of these chirps, and the other chirp will miss this coefficient. Moreover, the detection step may miss some signal coefficients. Consequently, the gathering process must be designed to be tolerant enough to be able to link chirplets that are non-consecutive in time.

Finally, some chirplet coefficients may be wrongly selected, due to noise induced false alarms, or interferences between the crossing chirps. Such a chirplet will not be gathered into the signal's chirp and will lead to a single false alarm chirplet. Therefore, in a postprocessing step, we remove chirps of length equal to the length of a single chirplet, in order to remove these false alarms.

1) Choice of the parameters: There are two sets of parameters that must be defined: the first one, related to the chirplet transform, and the second one related to the gathering into chirps. In addition, a probability of false alarm to determine the detection threshold is required.

The chirplet transform is related to the Short Time Fourier Transform, with an additional parameter, the chirprate. Consequently, the choice of the window's length, the overlap between consecutive windows and the zero padding depends on the usual criteria related to the STFT.

As seen in section II-B, the key parameter to achieve good detection is the chirprate step $\Delta_{d}$, which must be less than or equal to twice $\Delta_{d 0}=\left(\sum \phi_{M}[n]\right)^{-2}$. Let us consider that a priori we are able to bound the signals' chirprates by the MCT maximum chirprate $d_{\max }$. With $d_{\max }$ fixed, reducing the redundancy of the chirplet transform requires us to reduce the number of different chirprates. In order to maintain $\Delta_{d} \leq 2 \Delta_{d 0}$ we must choose a small analysis window. On the other hand, a small window will have difficulties in dealing with crossing chirps, and will detect more chirplets than longer windows, leading to a more error prone gathering step.

Experience with signals provided by the Defence Science and Technology Laboratory (DSTL) led to a choice of $M=4095$ and a maximum chirprate $d_{\max }=2.10^{-6}$. For these parameters a chirprate step satisfying $\Delta_{d} \leq 2 \Delta_{d 0}$ leads to 11 different chirprates. In addition, to minimize computation, we consider no overlap between consecutive windows or zero padding.

The parameters of the chirp gathering, the three thresholds $\delta_{n}, \delta_{k}$ and $\delta_{d}$, are chosen with respect to the parameters of the chirplet transform. A correctly detected chirplet associated with one of the signals chirps will have a chirprate close to the signal's true chirprate within the resolution of the chirprate discretization. The chirprate threshold is therefore set to the chirprate discretization size with a small tolerance factor, $\delta_{d}=$ $1.5 \Delta_{d}$.

${ }^{1}$ Due to the sensitive nature of the signals we are unable to present our analysis of these signals here.
The detection step selects only a single coefficient at each time-frequency location. Consequently, some coefficients may be missing near crossing chirps. The time threshold, $\delta_{n}$, is the key parameter which allows the correct association of closely disconnected chirplets into a single chirp. To allow for chirp crossings we permit two consecutive chirplets to be missing by setting the time threshold to three times the distance between consecutive chirplets, $\delta_{n}=3 M$.

The frequency threshold $\delta_{k}$ is not set to the frequency resolution of the chirplet transform. We want to be able to gather chirplets distant up to $\delta_{n}$ from the chirp. Given the uncertainty of the chirprate $\delta_{d}$, the furthest chirplet from a chirp candidate to the gathering with this chirp will have a frequency uncertainty equals to $\delta_{n} \delta_{d}$. This frequency uncertainty leads to a much higher frequency uncertainty than the frequency resolution of the transform. Consequently, we set the frequency threshold to match this uncertainty, that is $\delta_{k}=\delta_{d} \delta_{n}$.

The last parameter is probability of false alarm, to define a detection threshold. The chirplet's parameters lead to a correlation between the chirplet coefficient at a given timefrequency location, and thus will lead to a higher probability of false alarm than expected. Consequently, a low probability of false alarm is chosen, $p_{f a}=10^{-5}$.

\section{B. Results}

Figure 9 shows the result of the algorithm on a synthetic signal made of 6 signals containing 53 chirps of the same amplitude $A$. The SNR, defined as $10 \log _{10}\left(\frac{A^{2}}{\sigma^{2}}\right)$, is $-3 \mathrm{~dB}$. The chirplet is computed with a Gaussian window of 4095 points at 4096 computed frequencies. 11 discrete chirprates are computed, from $-2.10^{-6}$ to $2.10^{-6}$, such that the chirprate step $\Delta_{d}=4.10^{-7}$ is less than twice the critical chirprate step, $2 \Delta_{d_{0}} \approx 4.77 .10^{-7}$.

Among the 53 linear chirps, 9 are very short, less than 5000 points associated with a chirprate of very high absolute value. In order to maintain a manageable maximum chirprate these chirps are discarded and treated as discontinuities which are allowed in our signal model (2).

190 chirps are created from 3124 selected chirplets coefficients. All false alarm chirplets are isolated and are set in a single chirp. The post-processing removes the 146 chirps made of only one chirplet, leading to the correct detection of the 44 chirps existing in the analysed signal.

The errors on the estimated starting points and end points are all smaller than the window's length of the chirplet transform, and the error of the estimated frequency smaller than the frequency step. Thanks to the linear regression, the chirprates are very accurately estimated: the highest relative error on the chirprates is $0.15 \%$.

With the same parameters, the results are unchanged for SNR down to $-21 \mathrm{~dB}$.

\section{Extension: chirprate out of bounds}

The proposed algorithm supposes that the maximal chirprate of the signal's chirps is known. However, in certain prac- 

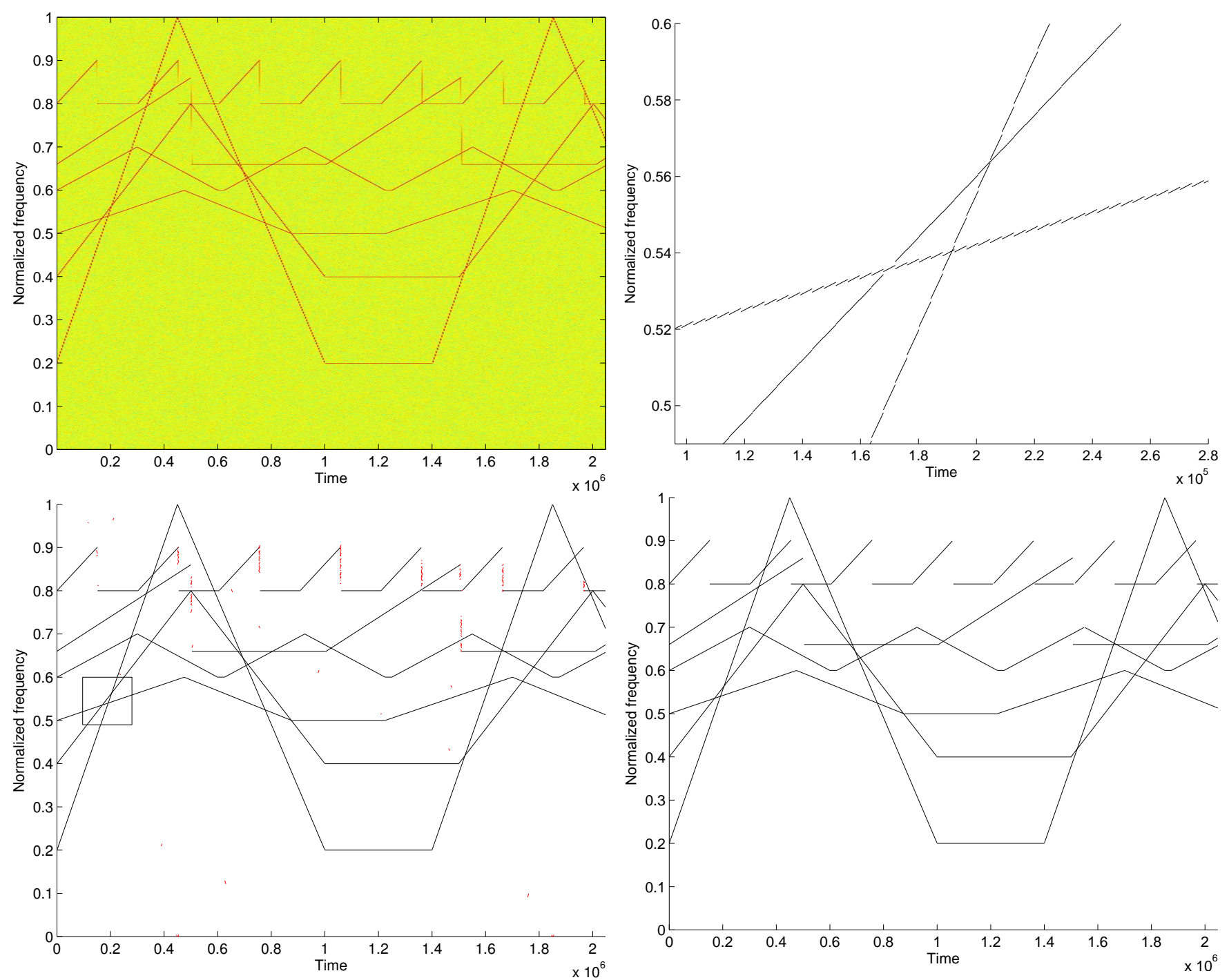

Fig. 9. Up left, spectrogram of the synthetic signal to analyse, made of 6 piece-wise linear signals of same amplitude. The SNR is $-3 \mathrm{~dB}$. Up right, zoom on the selected chirplets. Down left, results of the gathering of selected chirplets. False alarms are mainly located on very quickly decreasing chirps. The black box indicates the area of the zoom of the up right figure. Down right, the estimated chirps with the false-alarm chirps removed by the post-processing.

tical cases, the maximal chirprate of the signal being analyzed may not be known and may be outside of the range $\left[-d_{\max },+d_{\max }\right]$. Two problems then occur. First, according to the approximation (31), the energy obtained by a chirplet of chirprate $d_{\max }$ from a signal with a chirprate $d_{1}>d_{\max }$ will be reduced by a factor $\frac{1}{\left|d_{0}-d\right|}$, and consequently is less likely to be detected in low SNR. Second, the gathering algorithm will not be able to create chirps with such a chirprate, due to the frequency condition (47).

If $d_{1}$ were known we could increase $d_{\max }$, at the cost of more chirprate parameters of the chirplet transform, and thus a more expensive computational cost. Here we consider a modification of the algorithm presented in (IV-A), that has the same computational cost and is able to gather the chirplets with chirprates outside of the expected bounds into chirps, at the cost of the lower ability to detect these chirplets.

Two modifications are necessary in order to enable the detection and estimation of chirps with extreme chirprates.
First, assume that we have a created chirp with a chirprate $d_{1}>d_{\max }+\delta_{d}$. Selected chirplet coefficients will not have a high enough chirprate to fulfil the chirprate condition (46). Instead we propose a new chirprate condition that applies a saturation on the chirp's chirprate:

$$
\left|d-\max \left(-d_{\max }, \min \left(d_{\max }, d_{r}\right)\right)\right| \leq \delta_{d}
$$

The second problem is that a chirp whose chirprate is outside of the range $\left[-d_{\max },+d_{\max }\right]$ is impossible to create since a new chirp's estimated chirprate is taken from a chirplet and cannot correspond to its actual chirprate. This leads to the wrong frequency condition. A more flexible frequency condition for chirplets with extreme chirprates is given by:

$$
\begin{aligned}
d_{r}=d_{\max } & \& k_{i}+d_{r}\left(n-n_{i}\right)>k-\delta_{k} \\
d_{r}=-d_{\max } & \& k_{i}+d_{r}\left(n-n_{i}\right)<k+\delta_{k}
\end{aligned}
$$

Using this new frequency condition allows us to match chirplets whose chirprate are limited to the range $\left[-d_{\max }, d_{\max }\right]$ to newly created chirps. Grouping a second 

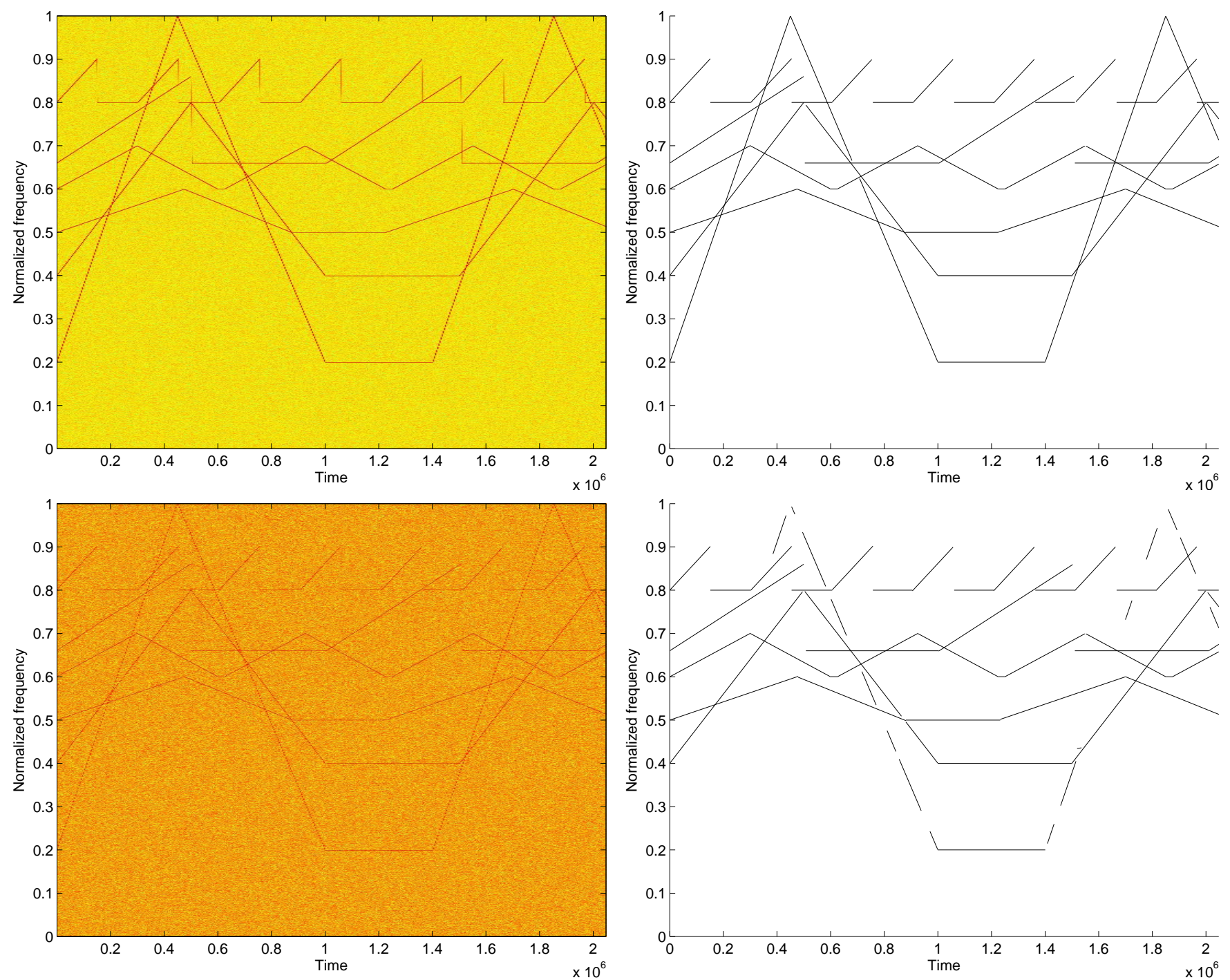

Fig. 10. Results for the gathering the selected chirplets with the extended algorithm (section IV-C) in the extreme case using only 3 distinct chirprates, $-4.10^{-7}, 0,4.10^{-7}$, and a maximum MCT chirprate much less than the actual signal chirprates $\left(2.10^{-6}\right)$. Top row, SNR is $-3 \mathrm{~dB}$ : results are similar to those in figure 9. Lower row, SNR is $-21 \mathrm{~dB}$. As expected, chirplets with extreme chirprates are poorly detected and consequently oorly gathered into chirps. Others chirps are not affected.

chirplet permits the estimation step to determine the relevant chirprate outside of the range $\left[-d_{\max },+d_{\max }\right]$. This new frequency condition allows the creation of a new chirp with an appropriate chirprate, but it cannot be used to associate a new chirplet to an existing chirp whose chirprate is out of range. In this case the old frequency condition (47) is appropriate To this end we only apply the new frequency condition (50) to newly created chirps. For any chirp that is composed of more than one chirplet the old frequency condition is used. Moreover, a stricter time condition is coupled with the new frequency condition, to permit this gathering only for consecutive windows, and avoid grouping chirplets at a single time index, which could create a chirp with an infinite chirprate. However, new false alarms chirps made of two chirplets with very different frequencies may appear. The post processing is now set to remove chirps of size less than or equal to twice the chirplet's length, at the cost of the risk of removing very small signal's chirps.
A final issue concerns the MCT spectrum. A signal with a chirprate higher than $d_{\max }$ will have a MCT spectrum larger than the computed upper-bound spectral window (35). Therefore the upper-bound spectral window will be overly narrow and we can expect an increase in the selection of incorrect chirplets in the case of a high SNR.

Figure 10 shows the result of this new algorithm on the synthetic signal used in previous section. The maximal chirprate is now $d_{\max }=4 \cdot 10^{-7}$. Decreasing the maximal chirprate while keeping the same chirprate step allows us to use fewer different chirprates in the chirplet transform to achieve a good detection, in this case only 3 different chirprates. On top, the SNR is $-3 \mathrm{~dB}$. All chirplets coefficients are accurately detected, and the chirp gathering leads to the same result than the previous one. However, for a low SNR=-21dB shown on bottom, some chirps are poorly detected and thus do not lead to the correct chirps.

In summary, these changes permit, with the same parameters 
of chirplet transform, to gather chirplets into chirps with a chirprate out of the range $\left[-d_{\max }, d_{\max }\right]$, but at the cost of more false alarm chirps, and without improving the detection of chirplets with high chirprates.

\section{Gathering into signals}

The final step is to gather the chirps back into signals. Here, we assume that the detection and gathering into chirps lead to an accurate result, as for example shown on figure 9.

The signals are Frequency Modulation Continuous Wave signals, and thus are continuous. The end of a linear chirp belonging to a given signal should be close to the beginning of the signal's next chirp. However, a difficulty occurs when the signal includes very short chirps associated with a very extreme chirprates, which are not detected since the detected signal is no longer continuous.

The goal is to associate each chirp's end (except those located at the end of the signal) to the beginning of another chirp, with the constraint that the beginning of a chirp may be associated to only one chirp. To do this, we first compute all the distances $d_{i, j}$ between the end point $\left[n_{e j}, k_{e j}\right]$ of the $j^{t h}$ chirp and beginning point $\left[n_{b i}, k_{b i}\right]$ of the $i^{t h}$ chirp

$$
d_{i, j}=\sqrt{\frac{1}{N^{2}}\left(n_{e j}-n_{b i}\right)^{2}+\frac{1}{K^{2}}\left(k_{e j}-k_{e i}\right)^{2}},
$$

with $N$ and $K$ the number of time and frequency bins, respectively. The normalisation is used to give the same weight to the time and frequency dimensions, independently from the chirplet transform's parameters. Each chirp $j$ is associated to the chirp $i$ leading to the smallest distance $d_{i, j}$.

It is possible to have a single chirp $j_{0}$ associated to several chirps $i_{k}$. In this case, we favour the connections between beginning and end points which are supposed to be strictly identical. This is done by keeping the connections such that the distance $d_{i, j}$ is below a threshold $t_{d}$

$$
t_{d}=\sqrt{\left(\frac{M}{N}\right)^{2}\left(M d_{\max }\right)^{2}}
$$

This threshold corresponds to the highest distance possible at a chirp transition, given the time uncertainty of length $M$.

All chirps which are still connected with a previous one are removed from the pool of available chirps, and a new timebased criterion is selected to connect the remaining chirps

$$
d_{i, j}^{\prime}=\left|n_{e j}-n_{b i}\right| \text {. }
$$

We assume here that the end of a chirp is close to the beginning of a new one, independently of their respective frequency. Given that the chirplets are quite small compared to the chirps' lengths, the probability that two chirps' end are detected at the same time bin are low.

Figure 11 illustrates the reconstructed signals. Each reconstructed signal is plotted in different colour, corresponding indeed to the different FMCW signals. Note that no information about the periodicity of the chirps, nor their chirprates have been used. Such information should however be useful to discriminate the signal if some ambiguity remains after the time criterion, and would also be important for classifying the type of radar.

This method avoids the expensive search for all possible combinations $(i, j)$ and selecting the result minimising all the distances.

\section{CONCLUSION}

We have presented in this paper an efficient algorithm for detecting and estimating linear chirps, in a context of LPI radars made of frequency modulation waves. First, the chirplet transform has been investigated, and an approximation proposed. A simpler time-frequency representation has been proposed, called Maximum Chirplet Transform (MCT), selecting the chirplet of highest energy at each time-frequency location. Using the approximation of the chirplet transform, the MCT spectrum of a chirp has been studied, leading to the definition of a window enclosing such a spectrum for any signal's chirprate.

The signal detection in the MCT has been proposed, with the goal of selecting as few relevant coefficients as possible. The chirp spectrum, centred on the highest energy points of the MCT spectrum is iteratively subtracted from this spectrum, until all spectrum points are below a detection threshold determined by a Neyman-Pearson approach. In this way, we do not need to recompute the MCT spectrum at each iteration, in contrast to MP techniques. This approach relies on the knowledge of the distribution of MCT coefficients containing noise only, which is known only when the chirplet coefficients at a given time-frequency location are uncorrelated. This correlation has been studied, and it has been observed that the coefficients are correlated when the chirprate step of the chirplet transform is low.

The second step of the algorithm gathers the detected coefficients into linear chirps with criteria based on time, frequency and chirprate, and estimates the chirp's parameters with a linear regression from the gathered coefficients. A postprocessing step removes "false alarm" chirps created with wrongly selected coefficients in the first step. This algorithm has been illustrated on a synthetic signal imitating 4 FMCW radars, leading to a very good reconstruction of the chirps for Signal-to-Noise Ratio down to $-21 \mathrm{~dB}$.

An extension of this algorithm have been proposed, allowing the detection of chirps with a chirprate out of the scope of the chirplet transform parameters, lowering the critical choice of the maximum chirprate of the chirplet transform. The main advantage is to reduce the importance of the choice of the maximum chirprate of the chirplet transform, and detecting unexpected chirps.

The detected chirps have been then gathered back into the FMCW signals constituting the analysed signal, using a criterion based on the time-frequency proximity of the starting and ending points of the detected chirps. This algorithm has shown very good results on the simulated data provided by the Defence Science and Technology Laboratory (agency of the United Kingdom Ministry of Defence).

We finally note that the underlying detection process could be easily adapted to incorporate other chirp profiles, e.g. 

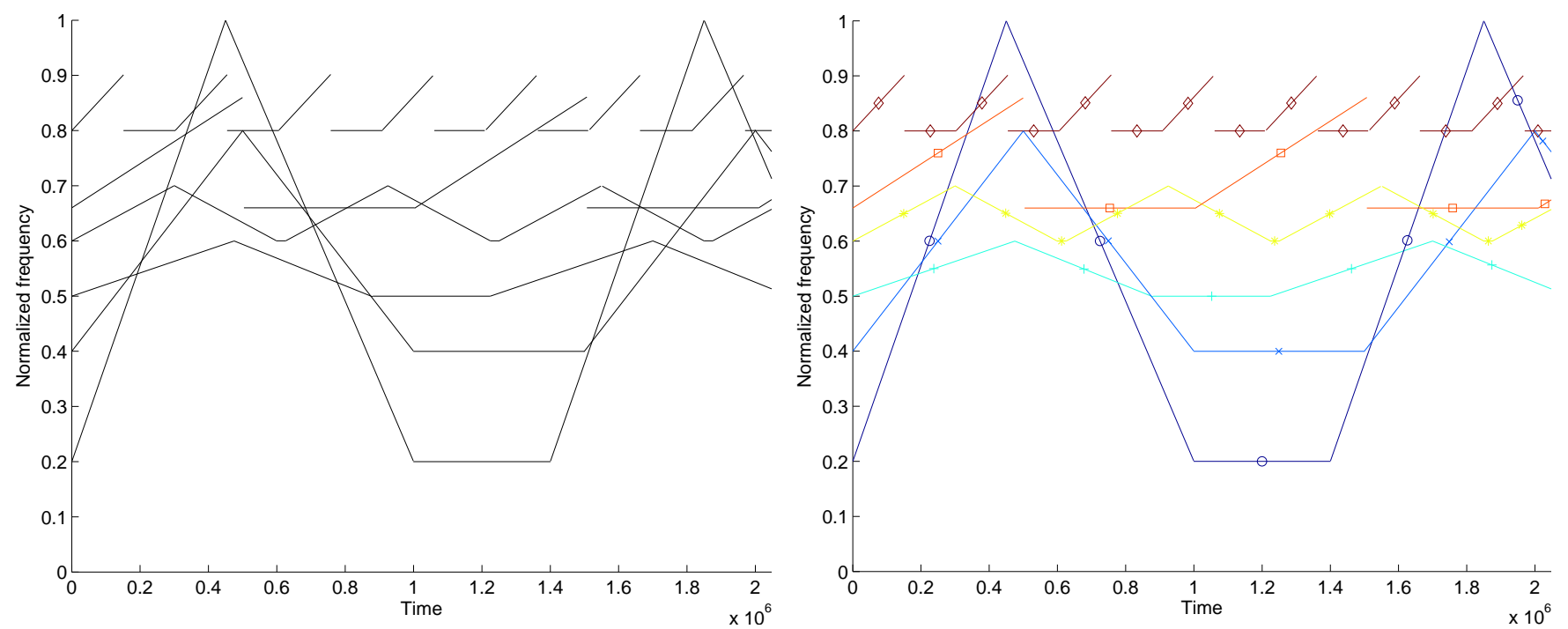

Fig. 11. Result of the gathering into signals of the chirps from figure 9. Each signal is correctly reconstructed, plotted in different colours.

quadratic chirps, as well as the detection and separation of pulse radar signals .

\section{REFERENCES}

[1] A. Denk, "Detection and jamming low probability of intercept (lpi) radars," Ph.D. dissertation, Naval Postgraduate School, 2006.

[2] S. Mann and S. Haykin, "The chirplet transform: A generalization of gabor's logon transform," in Proc. Vision Interface, Calgary, Canada, Jun. 1991.

[3] P. Leveau, L. Daudet, S. Krstulović, and R. Gribonval, "Model-based matching pursuit - estimation of chirp factors and scale of gabor atoms with iterative extension," in Signal Processing with Adaptative Sparse Structured Representations, Rennes, France, Nov. 2005.

[4] S. Brumby, K. Myers, and N. Pawley, "Capturing dynamics on multiple time scales: a multilevel fusion approach for cluttered electromagnetic data," in Proc. SPIE Multisensor, Multisource Information Fusion: Architectures, Algorithms, and Applications, vol. 7710, Orlando, Florida (US), 2010.

[5] A. Pai, E. Chassande-Mottin, and O. Rabaste, "Best network chirplet chain: Near-optimal coherent detection of unmodeled gravitational wave chirps with a network of detectors," Phys. Rev. D, vol. 77, no. 062005, pp. 1-22, 2008.

[6] E. J. Candès, P. R. Charlton, and H. Helgason, "Detecting highly oscillatory signals by chirplet path pursuit." Appl. Comput. Harmon. Anal., vol. 24, no. 1, pp. 14-40, 2008.

[7] B. Dugnol, C. Fernández, G. Galiano, and J. Velasco, "On a chirplet transform-based method applied to separating and counting wolf howls," Signal Process., vol. 88, no. 7, pp. 1817-1826, 2008.

[8] A. Petropulu, "Detection of multiple chirp signals based on a slice of the instantaneous higher-order moments," in Proc. Statistical Signal and Array Processing, Virginia BC, Canada, oct 1992.

[9] J. Dhanoa, E. Hughes, and R. Ormondroyd, "Simultaneous detection and parameter estimation of multiple linear chirps," in Proceedings of IEEE Conference on Acoustics, Speech and Signal Processing, Hong Kong, China, apr 2003.

[10] E. Chassande-Mottin and P. Flandrin, "On the stationary phase approximation of chirp spectra," in Proc. of the IEEE Int. Symp. on TimeFrequency and Time-Scale Analysis, Pittsburgh (US), 1998, pp. 117120.

[11] A. Hein, Processing of SAR Data: Fundamentals, signal processing, interferometry. Berlin: Springer-Verlag, 2004.

[12] P. R. White and J. Locke, "The performance of a fractional fourier transform based detector for frequency modulated signals," in Sensor Signal Processing for Defence, September 2010.

[13] G. Casella and R. L. Berger, Statistical Inference, 2nd ed. Duxbury Resource Center, 2001.
[14] F. Millioz and N. Martin, "Estimation of a white Gaussian noise in the Short Time Fourier Transform based on the spectral kurtosis of the minimal statistics: application to underwater noise," in Proceedings of IEEE Conference on Acoustics, Speech and Signal Processing, Dallas, Texas, USA, Mar. 2010.

[15] A. O'Hagan and J. Forster, The Advanced Theory of Statistics, Vol. 2B: Bayesian Inference, 2nd ed. Wiley, April 2004. 